Revue d'histoire de l'Amérique française

Q4. REVUE D'HISTOIRE DE L'AMÉRIQUE FRANÇAISE

\title{
Des eaux indésirables : Montréal et ses eaux de surface, 1796-1840
}

\section{Dany Fougères}

Volume 60, numéro 1-2, été-automne 2006

L'histoire environnementale

URI : https://id.erudit.org/iderudit/014596ar

DOI : https://doi.org/10.7202/014596ar

Aller au sommaire du numéro

\section{Éditeur(s)}

Institut d'histoire de l'Amérique française

ISSN

0035-2357 (imprimé)

1492-1383 (numérique)

Découvrir la revue

Citer cet article

Fougères, D. (2006). Des eaux indésirables : Montréal et ses eaux de surface, 1796-1840. Revue d'histoire de l'Amérique française, 60(1-2), 95-124.

https://doi.org/10.7202/014596ar
Résumé de l'article

Cet article retrace le passage à une nouvelle conception de l'aménagement du territoire municipal montréalais où les eaux de surface, d'abord perçues comme autant d'éléments et de phénomènes naturels et incontournables, peuvent et doivent dorénavant être chassées et évacuées, voire cachées (à l'intérieur de canalisations). Ce passage se produit au cours des premières décennies du $\mathrm{XIX}^{\mathrm{e}}$ siècle, au moment où les préoccupations en matière de salubrité urbaine s'additionnent aux préoccupations en matière de circulation des gens et des biens. Ce passage est réalisé avec l'établissement d'infrastructures urbaines et la construction de travaux publics et tient de l'action publique locale. À terme, ces ouvrages qui visent à contrôler et à faire disparaître les eaux de surface permettent de poser un nouveau regard sur l'environnement de la ville mais imposent, en même temps, une nouvelle réalité environnementale.
Tous droits réservés @ Institut d'histoire de l'Amérique française, 2006
Ce document est protégé par la loi sur le droit d'auteur. L'utilisation des services d’Érudit (y compris la reproduction) est assujettie à sa politique d'utilisation que vous pouvez consulter en ligne.

https://apropos.erudit.org/fr/usagers/politique-dutilisation/ 


\title{
Des eaux indésirables :
}

Montréal et ses eaux de surface, 1796 -1840

\author{
DANY FOUGÈRES \\ INRS - Urbanisation, Culture et Société
}

RÉSUMÉ - Cet article retrace le passage à une nouvelle conception de l'aménagement du territoire municipal montréalais où les eaux de surface, d'abord perçues comme autant d'éléments et de phénomènes naturels et incontournables, peuvent et doivent dorénavant être chassées et évacuées, voire cachées (à l'intérieur de canalisations). Ce passage se produit au cours des premières décennies du XIXe siècle, au moment où les préoccupations en matière de salubrité urbaine s'additionnent aux préoccupations en matière de circulation des gens et des biens. Ce passage est réalisé avec l'établissement d'infrastructures urbaines et la construction de travaux publics et tient de l'action publique locale. À terme, ces ouvrages qui visent à contrôler et à faire disparaître les eaux de surface permettent de poser un nouveau regard sur l'environnement de la ville mais imposent, en même temps, une nouvelle réalité environnementale.

ABSTRACT - This article describes the transition to a new conception of municipal land use planning in Montreal, in which surface water, initially viewed as an unavoidable natural phenomenon, became seen as something that could and should be flushed out and drained away, even hidden (inside pipes). This transition occurred during the first decades of the $19^{\text {th }}$ century, at a time when public health concerns were compounded by issues related to the

1. Cet article s'inspire en partie de données recueillies dans le cadre d'un travail doctoral réalisé à l'INRS-Urbanisation, Culture et Sociétés. Certaines informations sont présentées avec force détails dans Dany Fougères, L'approvisionnement en eau à Montréal. Du privé au public, 1796-1865 (Sillery, Septentrion, 2004). Enfin, nous avons bénéficié du concours financier de la Chaire de recherche du Canada en histoire environnementale du Québec $\mathrm{XIX}^{\mathrm{e}}-\mathrm{xx}^{\mathrm{e}}$ siècles de l'Université $\mathrm{du}$ Québec à Trois-Rivières pour la rédaction de cet article. 
movement of people and goods. Thanks to local public action, this transition was achieved through the development of urban infrastructures and public works. In the end, the work done to control and get rid of surface water provided a new way of looking at the urban environment, at the same time that it imposed a new environmental reality.

A U TOURNANT DU XIX ${ }^{\mathrm{e}}$ SIÈCLE, les eaux de surface provenant des cours d'eau intérieurs et des eaux de pluie et de fonte des neiges déterminaient en partie les conditions d'occupation du territoire urbanisé de Montréal. À cette époque, le fait d'être localisé à proximité d'un cours d'eau constituait encore un avantage pour certains Montréalais. C'était notamment le cas pour les chapeliers et les tanneurs. Toutefois, dès 1817, ces derniers se virent obligés de concentrer leurs activités à «l'Islette devant le port ${ }^{2}$ », preuve que certaines activités productives rejetaient des eaux que l'on voulait déjà soustraire à la vue et à l'odorat des Montréalais. Éléments attractifs pour les uns, les cours d'eau pouvaient, au contraire, ne s'avérer d'aucun intérêt pour certains riverains et passants ou, pire, constituer de sérieux irritants. En effet, pour certains, ils représentaient des obstacles naturels et imposaient des détours. Ils occasionnaient aux riverains des jours, voire des semaines d'inconfort chaque année, lors de la montée du niveau des eaux au moment de la fonte des neiges ou lors de pluies torrentielles. Les cours d'eau chargés de matières polluantes et nauséabondes suscitaient aussi la crainte que des maladies se propagent. Les Montréalais allaient graduellement considérer que les eaux de pluie et les eaux de la fonte des neiges, ces eaux qui «tombaient du ciel», devaient être chassées parce qu'elles entravaient la libre circulation, détruisaient la fondation des rues et répandaient la maladie ${ }^{3}$.

Cet article retrace le passage à une nouvelle conception de l'aménagement du territoire municipal : les eaux de surface, d'abord perçues comme autant d'éléments et de phénomènes naturels et incontournables, peuvent et doivent dorénavant être chassées et évacuées, voire cachées (à l'inté-

2. Règles et règlements de Police, pour les Fauxbourgs et la Cité de Montréal (Montréal, James Lane, 1817), article 34. Sur les activités de transformation avant le $\mathrm{XIX}^{\mathrm{e}}$ siècle et leurs liens avec l'accès aux cours d'eau, voir Chloé Deligne, Bruxelles et sa rivière. Genèse d'un territoire urbain (12 $12^{\mathrm{e}}-18^{\mathrm{e}}$ siècle) (Turnhout, Belgique, Brepols Publishers, 2003); André Guillerme, Les temps de l'eau. La cité, l'eau et les techniques. Nord de la France fin III - début XIX ${ }^{\mathrm{e}}$ siècle (Paris, Champ Vallon, coll. "Milieux", 1983).

3. La présence des eaux de surface sur l'espace public pouvait sans doute affecter de manière considérable certaines propriétés privées. Nous n’avons pas étudié cette question qui a certes pu influencer les administrateurs locaux. 
rieur de canalisations). Ce passage se produit au cours des premières décennies du $\mathrm{XIX}^{\mathrm{e}}$ siècle et tient de l'action publique locale. S'il revêt des caractéristiques particulières à Montréal en raison des spécificités de son territoire, il ne lui est pas unique puisque les grandes villes du monde occidental travaillent à la même époque à chasser leurs eaux de surface ${ }^{4}$, pour ne conserver que les fonctions édilitaires et monumentales de l'eau.

Ainsi, nous verrons qu'entre 1796 et 1840, on assiste en quelque sorte à un renouvellement de la rencontre entre les possibilités et les contraintes qu'offre l'environnement aux Montréalais. Ce renouvellement est dicté par de nouvelles considérations territoriales émises en $1796^{5}$ par le pouvoir colonial et appuyé par des ambitions locales de circulation et de salubrité urbaine. C'est dans le cadre de ce renouvellement que s'inscrivent le contrôle et la disparition des eaux de surface dans la ville, réalisés par l'établissement d'infrastructures urbaines et la mise en ouvre de travaux publics ${ }^{6}$. En d'autres termes, grâce à la confection d'ouvrages techniques, on procède à une reconfiguration de l'environnement naturel du territoire municipal montréalais, ce qui, à terme, aura pour résultat la disparition des eaux de surface dans la ville.

Plusieurs études ont déjà révélé les enjeux économiques, sociopolitiques et sanitaires entourant le déploiement d'infrastructures urbaines (aqueduc, routes, égouts) ${ }^{7}$. Il reste encore à inscrire davantage l'étude de ces infrastructures dans une série de rapports et d'échanges spatiaux, d'une part, en analysant les conditions du territoire rendant possible (ou nécessaire) leur implantation et, d'autre part, en identifiant les avantages et les contraintes que ces infrastructures offrent ou imposent, une fois établies, sur l'environnement urbain et sur les activités de ses habitants. L'établissement d'infrastructures urbaines permet de poser un nouveau regard sur l'environnement de la ville mais celles-ci imposent également une nouvelle

4. Voir sur le passage de l'eau qui devient progressivement indésirable dans la ville, André Guillerme, Les temps de l'eau..., op. cit.

5. Statuts du Bas-Canada, 36 Geo III (1796) chapitre 9. En partie pour répondre à des questions d'organisation politique et financière, la loi des chemins s'inscrivait aussi dans une perspective d'aménagement du territoire. Elle visait à "produire de l'ordre».

6. Sur les liens entre infrastructures et environnement, voir notamment C. M. Rosen et J. A. Tarr, "The Importance of an Urban Perspective in Environmental History», Journal of Urban History, 20,3 (mai 1994): 299-310.

7. Pour l'expérience canadienne, voir C. Armstrong et H. V. Nelles, Monopoly's Moment. The Organization and Regulation of Canadian Utilities, 1830-1930 (Philadelphie, Temple University Press, 1986); N. R. Ball, dir., Bâtir un pays. Histoire des travaux publics au Canada (Montréal, Boréal, 1988). 
réalité environnementale comme l'a bien démontré $\mathrm{M}$. Melosi ${ }^{8}$ pour l'expérience américaine ainsi que $\mathrm{K}$. Bowie ${ }^{9}$ et $\mathrm{F}$. Caron ${ }^{10}$ pour l'expérience française. Le présent article vise aussi à contribuer à cette réflexion sur la dynamique des rapports entre territoire et infrastructures.

La disparition des eaux de surface à Montréal trouve en partie sa légitimité dans l'accroissement de la population ${ }^{11}$ et des activités urbaines mais elle est également freinée par les limites financières et techniques de l'administration locale et des travaux publics ${ }^{12}$. Quoi qu'il en soit, nous verrons qu'aux alentours des années 1840 , le renouvellement de l'environnement urbain est bien enclenché, même si les réalisations qui l'accompagnent (infrastructures techniques en particulier) ne sont pas toutes complétées ou même commencées. L'histoire que nous présentons ici en est une de rencontres entre le monde idéel ou culturel et le monde matériel et s'exprime sur le terrain de la réalité environnementale d'un espace en période de développement ${ }^{13}$.

D'abord, nous dressons un inventaire sommaire des types d'eaux de surface que nous retrouvons à Montréal au tournant du XIX ${ }^{\mathrm{e}}$ siècle ${ }^{14}$. Par

8. Martin V Melosi, The Sanitary City. Urban Infrastructure in America from Colonial Times to the Present (Baltimore, The Johns Hopkins University Press, 2000).

9. Karen Bowie, dir., La modernité avant Haussmann: formes de l'espace urbain à Paris, 1801-1853 (Paris, Éditions Recherches, 2001).

10. François Caron, dir., Paris et ses réseaux: naissance d'un mode de vie urbain $X I X^{\mathrm{e}}-\mathrm{XX} \mathrm{X}^{\mathrm{e}}$ siècles (Paris, Bibliothèque historique de la Ville de Paris, 1990).

11. Entre 1796 et 1840 , la population de Montréal passe de 8000 à 9000 habitants à tout près de 50000 habitants. Le nombre de maisons est multiplié par cinq.

12. Entre 1796 et 1840 , les dépenses en matière de travaux publics sont multipliées par onze pour atteindre $6000 £$. En 1843, soit trois ans après la mise en place de la Corporation municipale (administrée par des élus) les mêmes dépenses dépassent les $18000 £$. En 1817, on dénombre 136 rues à Montréal; en 1842, on en dénombre 199 et le kilomètre linéaire total de celles-ci atteint 84 kilomètres.

13. Le concept d'environnement n'existait pas au $\mathrm{xIX}^{\mathrm{e}}$ siècle. Comme nous le savons, aujourd'hui celui-ci fait référence à différentes réalités, par exemple, le territoire lui-même, la qualité de l'air, des sols, etc., qui ici réfèrent à des questions davantage liées aux problématiques de consommation, préservation et pollution. Dans le cas qui nous intéresse, le terme qui pourrait le plus se rapprocher du concept d'environnement est celui de salubrité urbaine qui, en définitive, fait référence à la propreté de la ville. La salubrité urbaine au $\mathrm{xIx}^{\mathrm{e}}$ siècle est un concept ou une réalité à mi-chemin, en quelque sorte, des concepts actuels de pollution urbaine, de santé et de sécurité publique. La salubrité urbaine ne réfère pas à proprement parler aux questions d'hygiène, lesquelles faisaient davantage référence à la dimension domestique (les résidences, l'habitat). Dans son livre, Martin V. Melosi présente l'évolution des rapports entre les concepts de salubrité urbaine, d'environnement et d'écologie: Martin V. Melosi, The Sanitary City. Urban Infrastructure..., op. cit.

14. Ces eaux de surface sont encore si nombreuses, imposent des contraintes si fortes et dictent à ce point les conditions d'usage du territoire montréalais qu'il nous viendrait à l'idée de parler de Montréal ville d'eaux: non pas parce que Montréal est une ville qui exploite la ressource hydrique en soi mais bien parce que la présence des eaux de surface rythme le quotidien des Montréalais. 
la suite, nous portons notre analyse sur les rivières et les ruisseaux qui se trouvaient dans la ville, au cœur du territoire urbanisé. Nous terminons notre étude en traitant des eaux de pluie et de fonte des neiges.

\section{INVENTAIRE DES EAUX DE SURFACE DANS LA VILLE}

À l'époque où le territoire habité de Montréal se limitait à l'actuel secteur du Vieux-Montréal et de ses environs immédiats, les cours d'eau étaient partie intégrante du quotidien des Montréalais. En effet, outre le fleuve Saint-Laurent ${ }^{15}$ qui s'offrait majestueusement devant la ville, des cours d'eau d'envergure mineure entouraient Montréal et traversaient ou serpentaient les faubourgs qui étaient toujours séparés de cette dernière par ses fortifications en 1800. Grâce au plan de ville dressé par Louis Charland en 1801 (figure 1), il est possible de localiser ces cours d'eau.

Le plan de Charland indique, du côté nord de la vieille ville, la présence du ruisseau Saint-Martin qui coulait d'est en ouest, pour aller à la rencontre du ruisseau Prud'homme et de la Petite Rivière. Également appelée rivière Saint-Pierre, la Petite Rivière venait du sud-ouest et se jetait dans le fleuve Saint-Laurent à la hauteur de la Pointe-à-Callière, après avoir reçu les eaux du ruisseau Prud'homme et du ruisseau Saint-Martin.

Aussi, d'autres cours d'eau s'écoulaient en périphérie du territoire habité de 1801 mais la carte de Charland ne permet pas de les situer. Dans son rapport sur les chemins de 1840, Jacques Viger, alors inspecteur des chemins pour la municipalité, en mentionne l'existence. Situés à la périphérie des zones urbanisées de 1801, ces cours d'eau se trouvaient maintenant en totalité ou en partie à l'intérieur de zones urbanisées. La taille des faubourgs s'était en effet considérablement accrue en l'espace de quarante ans. L'inventaire de Viger se présentait comme suit:

Celui du faubourg Québec, se déchargeant rue Craig; 2. Celui du faubourg St. Louis, pied de la côte à Barron, se déchargeant rue St. Laurent; 3. Celui du faubourg St. Laurent, pied de la côte à Barron se déchargeant sur St. Charles Borromée; 4. Ceux du faubourg St. Antoine, dont un vient de la Montagne, et l'autre sort du canal ou Tunnel de la rue Craig, se déchargeant tous les deux au pont Prud'homme ; 5. Enfin, celui du faubourg St. Joseph, venant du Fief de ce nom, se déchargeant au pont Prud'homme. Tous ces cours-d'eau, à l'exception d'un seul peut-être (celui sortant de la rue Craig), ont besoin d'être nétoyés dans leurs lits et plus ou moins recalés, tous les ans ${ }^{16}$.

15. Comme son titre l'indique, cet article ne traite pas du fleuve Saint-Laurent. De même, les problèmes d'inondations dues au fleuve ne sont pas étudiés ici.

16. Jacques Viger, Rapports sur les chemins, rues, ruelles et ponts de la Cité et Paroisse de Montréal, Avril et Mai 1840 (Montréal, Imprimé par John Lovell, 1841), 12. 
Figure I

Montréal en I80I, d'après le plan de Louis Charland

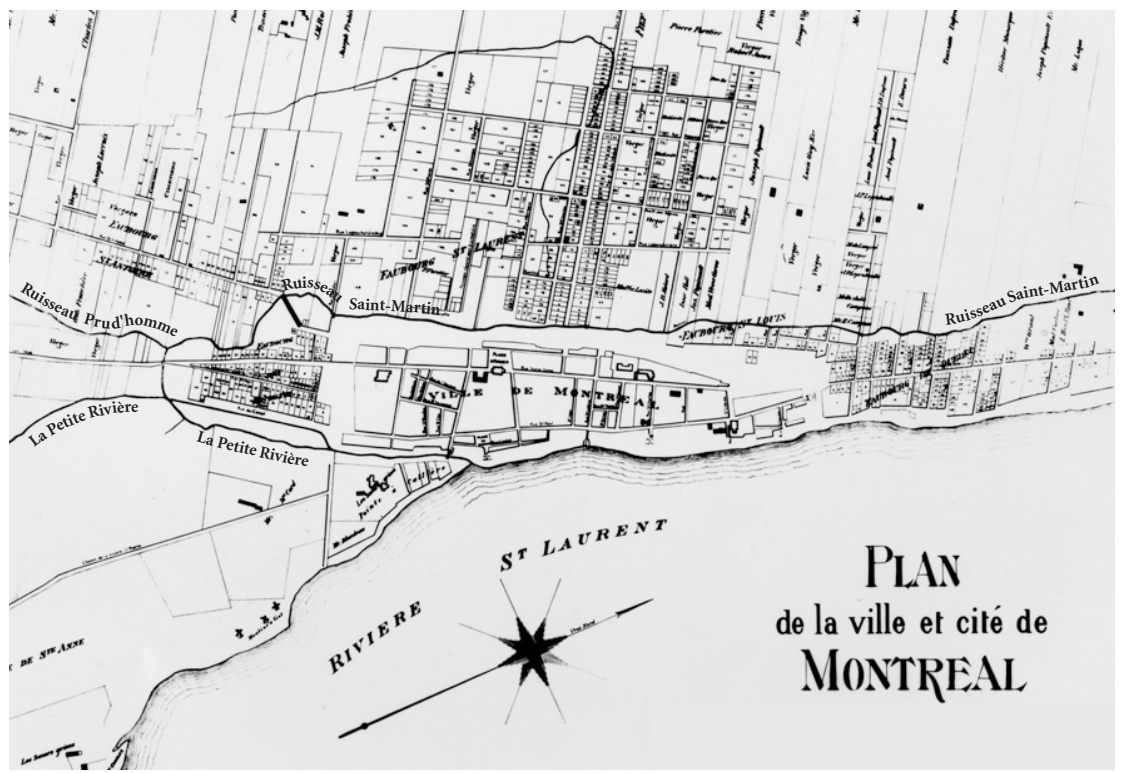

Source: Ville de Montréal, Gestion des documents et archives (VM-GDA), VM-66, Fonds Collection des cartes et plans. Les indications sont de l'auteur, voir Dany Fougères, L'approvisionnement en eau à Montréal..., op. cit., 214.

Les archives municipales font également mention du ruisseau Mignon traversant la rue Saint-François-Xavier, d'un ruisseau traversant la rue Lagauchetière et d'un autre ruisseau venant de la montagne par les rues Prévost et Guy (traversant cette dernière ${ }^{17}$. Cet inventaire n'a pas la prétention d'être exhaustif, mais nos recherches nous permettent de croire que nous avons là l'essentiel des cours d'eau qui se trouvaient sur le territoire urbanisé de l'époque.

Les eaux de pluie et les eaux de fonte des neiges faisaient partie du quotidien montréalais. En effet, en raison d'infrastructures et de travaux publics qui restaient encore largement rudimentaires à la fin du $\mathrm{XvIII}^{\mathrm{e}}$ siècle et au début du $\mathrm{xIx}^{\mathrm{e}}$ siècle $^{18}$, ces eaux s'accumulaient sur les sols et pou-

17. Ville de Montréal, Gestion des documents et archives (VM-GDA), VM-35, Fonds Juges de paix de Montréal (FJPM), Procès-verbaux des Sessions spéciales de la paix, 27 août 1796, 8-9; 16 septembre 1826, 69; 5 avril 1820, 247.

18. Voir Dany Fougères, «Des rues et des hommes : les commencements des politiques publiques locales en matière de travaux publics. Montréal, 1796-1840», Scientia Canadensis, 25 (2001): 31-65 ; J. A. Tarr, "The Evolution of the Urban Infrastructure in the Nineteenth and Twentieth Centuries", dans R. Hanson, Perspectives on Urban Infrastructure (Washington, D.C., National Academy Press, 1984), 4-66. 
vaient y demeurer pendant plusieurs heures, voire plusieurs jours. Au rythme des saisons, elles prenaient du volume, si l'on peut dire, et se chargeaient de matières polluantes, ce qui pouvait devenir problématique au moment où s'accentuaient la densité d'occupation des sols ainsi que les déplacements des personnes et des biens. En l'absence de travaux publics d'envergure (voire la construction d'un réseau de conduites d'égout), une des façons de "contrôler» le ruissellement de ces eaux consistait à tracer tout un circuit de rigoles et de fossés. Les eaux de la fonte des neiges étaient importantes au $\mathrm{XIX}^{\mathrm{e}}$ siècle, car la neige qui s'accumulait dans la ville n'était pas "ramassée» comme aujourd'hui mais "tapée ${ }^{19}$ ". La figure 2 montre une rue de Montréal dans les dernières décennies du $\mathrm{XIX}^{\mathrm{e}}$ siècle : on $\mathrm{y}$ voit des voitures surélevées par l'accumulation de la neige et plus bas, des marcheurs sur les trottoirs.

Figure 2

\section{La neige «tapée » sur les rues}

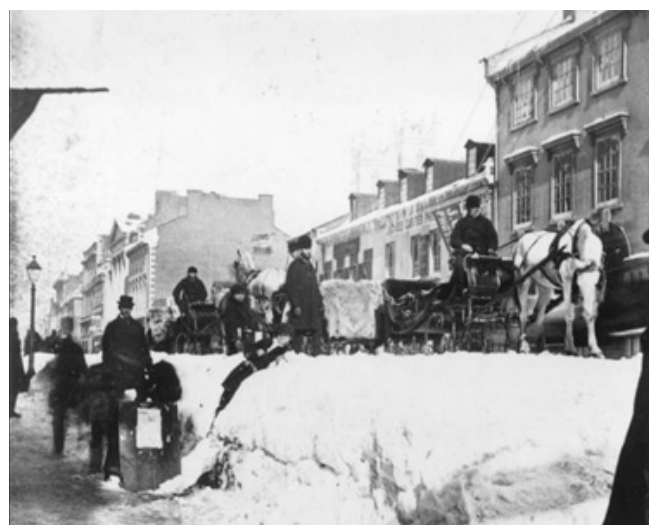

Source: Archives et Bibliothèque nationales du Canada

\section{LES COURS D'EAU}

Dans la première moitié du $\mathrm{xIx}^{\mathrm{e}}$ siècle, l'eau était donc omniprésente à Montréal. Comme dans les autres villes d'Amérique et d'Europe à la même époque, un des fondements premiers de l'action municipale menée par les administrateurs locaux (les juges de paix $^{20}$ ) fut d'assurer et d'accroître la

19. Pour l'expérience des autres villes canadiennes, voir N. R. Ball, dir., Bâtir un pays..., op. cit.

20. Statut du Bas-Canada, 36 Geo III, 1796. La loi des chemins de 1796 mettait en place une autorité locale, celle des juges de paix. Ces derniers étaient nommés par le pouvoir colonial afin de veiller uniquement et exclusivement à l'administration de la ville (la ville fortifiée et les faubourgs). 
circulation des gens et des biens: «Désenclaver les villes, mettre en contact les uns avec les autres les espaces productifs est le maitre mot de l'époque», ainsi que l'écrit F.-X. Merrien ${ }^{21}$. Donc, en un mot : circulez!

Mais encore fallait-il pour cela contrer, ou à tout le moins parvenir à minimiser, l'importance de ces obstacles que représentaient les cours d'eau et maîtriser, voire parvenir à évacuer, les eaux de pluie et de fonte des neiges. En somme, des défis à la fois singuliers à Montréal de par la spécificité de son territoire mais aussi communs à toutes les villes de l'époque parce que cette nécessité de contrôler les eaux était vécue par chacune d'elles: d'abord pour des besoins de circulation ${ }^{22}$ puis, rapidement, pour des considérations de salubrité urbaine ${ }^{23}$.

Le nettoyage des rues et le ramassage des déchets sont des activités directement liées aux questions de salubrité urbaine. À l'époque, elles permettaient aussi d'assurer la libre circulation des gens et des biens. À preuve, les délibérations des juges de paix sont remplies de cas où l'on demande d'enlever les obstructions sur les rues, où l'on se plaint des bois et matériaux laissés ici et là sur la voie publique, où l'on recherche les propriétaires de chevaux morts étendus sur le pavé et, faute d'y parvenir, où l'on se résigne à enlever la bête aux frais du public.

\section{Circulez! Circulez! Des obstacles à franchir}

La réalisation d'un réseau de rues étendu à l'échelle du territoire habité demandait du savoir-faire technique, des efforts financiers, un leadership politique et des assises juridiques. À cela s'ajoutait l'obligation de prendre en compte la réalité environnementale du territoire, sa réalité physique dont les éléments distinctifs d'un secteur à l'autre s'avéreront tour à tour occasions et contraintes, en fonction des connaissances scientifiques et techniques du moment. En somme, les décideurs du moment ne pouvaient qu'imaginer l'imaginable: les grands travaux de canalisations souterraines sur plusieurs kilomètres n'étaient pas encore concevables en 1790 ou en 1800 comme d'ailleurs, à titre d'exemple, la construction d'un

21. F.-X. Merrien, La Bataille des eaux. L'hygiène à Rennes au XIX ${ }^{\mathrm{e}}$ siècle (Rennes, Presses universitaires de Rennes, 1994), 80.

22. André Guillerme, «Le pavé de Paris», dans F. Caron et al, dir., Paris et ses réseaux..., op. cit., 59-82 ; J. A. Tarr, «Building the Urban Infrastructure in the Nineteenth Century: An Introduction», Infrastructure and Urban Growth in the Nineteenth Century (Chicago, Public Works Historical Society, Essays in Public Works History), 14 (décembre 1985): 61-85.

23. Martin V. Melosi, The Sanitary City. Urban Infrastructure in America..., op. cit.; Sabine Barles, La ville délétère. Médecins et ingénieurs dans l'espace urbain XVIII ${ }^{\mathrm{e}}$-XIX ${ }^{\mathrm{e}}$ siècle (Paris, Champ Vallon, coll. "Milieux», 1999); J. A. Peterson, "The Impact of Sanitary Reform Upon American Urban Planning, 1840-1890", Journal of Social History, 13 (1979): 83-104. 
pont suspendu sur plusieurs kilomètres. Déjà, dans la décennie 1840 par contre, une canalisation souterraine à l'échelle de la ville était possible du point de vue technique, bien que sa réalisation ait tardé pour des raisons financières, politiques et légales.

Comme toutes les villes du monde, Montréal n'était pas une plaine uniforme, libre de tout obstacle, composée indistinctement des mêmes attributs partout sur son territoire. Pour réaliser la mise en place d'un réseau de rues étendu à tout le territoire habité ${ }^{24}$, il fallait construire aussi des infrastructures "connexes » à la rue permettant d'assurer un tracé continu, sans rupture ou, à tout le moins, sans «interruption prolongée ${ }^{25}$ ». À Montréal, les administrateurs locaux durent s'attaquer à la présence des cours d'eau intérieurs qui serpentaient et traversaient le territoire urbanisé puisque ceux-ci représentaient des obstacles et même des "coupures" dans l'établissement d'un réseau de rues en continu. La loi leur reconnaissait à cet effet des pouvoirs et des responsabilités :

Et qu'il soit de plus statué par l'autorité susdite, que lorsque les fossés, canaux et cours d'eau qui ont été ci-devant faits (étant réparés, nettoyés et tenus ouverts par le travail imposé par cet Acte, ci-après autorisé) ne seront pas suffisants pour la décharge de l'eau qui sera sur les grands chemins ou dans les rues, alors et dans tout tel cas il sera et pourra être légal aux dits Inspecteurs ou Sous-inspecteurs par l'ordre au moins de deux Juges à Paix, d'ouvrir de nouveaux fossés, canaux et cours d'eau sur les terres et terreins joignant tels grands chemins ou rues, ou sur toute autres terre ou terrein (n'étant point un jardin ou verger) s'il est nécessaire pour faire écouler plus aisément et plus efficacement les eaux des dits grands chemins et rues, et aussi pour tenir tels canaux, fossés et cours d'eau nets et libres ${ }^{26}$.

Les procès-verbaux des réunions tenues par les dirigeants locaux et les rapports annuels de l'inspecteur des chemins ${ }^{27}$ révèlent l'existence de plus

24. Statuts du Bas-Canada, 36 Geo III (1796) chapitre 9, article 39. Les juges de paix étaient "nommés et autorisés comme il est ci-après pourvu, d'établir et de régler les grands chemins, rues et ponts dans les cités et paroisses susdites, dans lesquelles ils exerceront leur charge respectivement».

25. Comme nous le savons, la présence d'un pont permet de passer d'une rive à l'autre d'un cours d'eau. Toutefois, parce que plusieurs rues peuvent converger vers un seul pont, on observe parfois aux accès de celui-ci des ralentissements, voire des attentes. Donc, malgré la présence d'un pont, un cours d'eau représente toujours un obstacle à la fluidité du transport.

26. Statuts du Bas-Canada, 36 Geo III (1796) chapitre 9, article 42.

27. Statuts du Bas-Canada, 36 Geo III (1796) chapitre 9, article 40. La loi prévoyait la nomination d'un inspecteur des chemins. Ce dernier était en quelque sorte le premier (en ordre d'importance) fonctionnaire municipal. Il avait à mettre en œuvre les intentions et les projets dictés par les juges de paix. Avec Jacques Viger qui aura occupé ce poste pendant plus de vingt ans, l'expérience aura 
de soixante ponts différents dans Montréal entre 1800 et 1840 . Et ce nombre, bien que considérable, n'est qu'une approximation (même si nous l'estimons assez près du nombre total). Les archives indiquent que la réalisation de certains ponts érigés dans la ville nécessitait, pour l'époque, du savoir-faire et des investissements financiers importants. Certains d'entre eux mesuraient jusqu'à 17 mètres de long. Plusieurs étaient des ouvrages de maçonnerie ou étaient faits en bois mais s'appuyaient sur des piliers de pierre ou de brique.

Lors d'une réunion des juges de paix du mois d'août 1821, il est "Ordonné que l'inspecteur des chemins annonce l'érection d'un nouveau pont sur la Petite Rivière au bas de la rue Saint-Joseph de 30 X 20 pieds $^{28}$ ». Dans le faubourg Saint-Laurent, sur la rue Craig, les juges de paix approuvent quelques années plus tard la construction d'un «pont en bois sur quai en pierre, vis-à-vis les rues Saint-Urbain et Saint-Joseph» et un autre d'après les mêmes caractéristiques face aux rues Bleury et Saint-Pierre ${ }^{29}$. Sur la rue Bonaventure, à la rencontre des faubourgs Saint-Joseph et SaintAntoine, un pont en bois de 36 pieds de long sur piliers en pierre est construit pour traverser la Petite Rivière ${ }^{30}$. Autres exemples qui indiquent que la réalisation de certains ponts commandait du savoir-faire et des investissements importants: en juillet 1826, on demande aux maçons Redpath et McKay d'évaluer l'état du pont de pierre nommé Franchère à la Pointe-à-Callière, on annonce la réalisation de travaux de réparation sur le pont Charland et le pont Torrence et on décide de construire un "pont volant en bois » à l'embouchure de la Petite Rivière, à la Pointe-à-Callière, pourvu que le coût de sa construction n'excède pas $25 £^{31}$. Au tournant des années 1800 , la construction du pont en pierre Prud'Homme, sur la rivière du même nom dans le faubourg des Récollets, avait nécessité plus de deux ans de discussions ${ }^{32}$. Elle était évaluée à $100 £^{33}$ en 1799 , ce qui

montré que celui-ci était tout autant un exécutant qu’un initiateur de projets, cela sans doute grâce à la connaissance fine qu'il avait de l'environnement urbain (il était l'homme de terrain).

28. VM-GDA, VM-35, FJPM, Procès-verbaux des Sessions spéciales de la paix, 25 août 1821, 368.

29. VM-GDA, VM-35, FJPM, Procès-verbaux des Sessions spéciales de la paix, 9 mai 1829, 295.

30. VM-GDA, VM-35, FJPM, Procès-verbaux des Sessions spéciales de la paix, 27 juin 1834, 243.

31. VM-GDA, VM-35, FJPM, Procès-verbaux des Sessions spéciales de la paix, 3 juillet 1826, 4344.

32. VM-GDA, VM-35, FJPM, Procès-verbaux des Sessions spéciales de la paix, 7 mars 1799, 270; 8 juin $1799,96-97 ; 28$ juin 1800,$125 ; 29$ juillet 1800,127 . À la première réunion, on annonçait l'intention de construire le pont Prud'Homme. À la réunion suivante, on présentait un rapport sur son emplacement futur. Lors des réunions suivantes, on donnait avis public pour la consultation des plans du pont puis on informait l'assemblée qu'aucune opposition ne s'était manifestée face à la réalisation du projet.

33. VM-GDA, VM-35, FJPM, Procès-verbaux des Sessions spéciales de la paix, 4 mai 1799, 92. 
représentait un investissement colossal si l'on tient compte du fait que les dépenses annuelles totales de la ville s'élevaient à ce moment aux alentours de $500 £$.

La demande pour l'érection des ponts pouvait aussi venir de la population comme en témoigne cette requête de l'été 1802 par laquelle un groupe de seize pétitionnaires de la Côte-Saint-Paul demande de "construire un pont sur la rivière St. Pierre - chemin menant à Lachine ${ }^{34}$ ». Les ponts étaient partie intégrante du paysage montréalais des années 1800-1840 et en leur absence, c'étaient les déplacements des personnes et des biens qui en souffraient comme en témoigne cet extrait d’archives: «La rue Guy, au faubourg St. Joseph, se trouve impraticable; faute de bons ponts sur les ruisseaux qui la traversent: un d'eux est même levé, à ce que dit le piqueur ${ }^{35}$.»

Certes, les ponts construits sur le territoire montréalais n'étaient sans doute pas tous imposants. Plusieurs ne demandaient probablement que peu de savoir-faire technique et peu d'investissements monétaires pour leur construction et leur entretien. Ils ne devaient probablement traverser que de petits ruisseaux, peut-être même que des fossés creusés au croisement de deux rues. Tout de même, quels que soient leur dimension et les obstacles à franchir, leur nombre impressionnant indique deux choses: dans la première moitié du $\mathrm{XIx}^{\mathrm{e}}$ siècle, Montréal était une ville d'eaux et on tenta d'abord de passer par-dessus ces eaux, plutôt que de s'en soustraire en les faisant disparaître à l'intérieur de canalisations souterraines.

Circulez était certes le mot d'ordre émis en 1796 et repris par les dirigeants locaux. Bien que l'analyse exhaustive du caractère aménagiste de la loi des chemins reste à faire, ses dispositions permettent d'arriver à ce constat et d'y inscrire dans le même esprit les travaux visant à contrer l'obstacle de l'eau. L'esprit général du concept moderne de l'aménagement, soit celui de "produire de l'ordre ", était bien présent en $1796^{36}$ : subdiviser le territoire urbain en districts, dresser un plan de ville, annoncer les projets futurs $^{37}$, identifier et reconnaître le réseau de rues, en standardiser la

34. VM-GDA, VM-35, FJPM, Procès-verbaux des Sessions spéciales de la paix, 17 juillet 1802, 156157. La rivière Saint-Pierre était l'autre nom donné à la Petite Rivière. Ce «chemin menant à Lachine» auquel faisaient mention les pétitionnaires n'était pas construit. La construction du pont, selon les mêmes pétitionnaires, était une condition à sa construction.

35. VM-GDA, VM-35, FJPM, Rapports et dossiers, $\mathrm{n}^{\circ}$ 3-30-20-VM1, $5^{\mathrm{e}}$ dossier 1810-1-1, Rapport de l'Inspecteur des chemins concernant l'état des rues de la ville et autres objets, 14 septembre 1821.

36. La loi portait, d'une part, sur le territoire bas-canadien en général (articles 1 à 38) et, d'autre part, sur les grandes villes de la colonie qu'étaient Québec et Montréal (articles 39 à 80).

37. Statuts du Bas-Canada, 39 Geo III, 1799, chapitre 5, Acte qui amende un acte passé dans la trente-sixième année du règne de sa présente Majesté, intitulé, "Acte pour faire, réparer et changer les chemins et ponts dans cette province, et pour d'autres fins", article 27. 
largeur, détourner des cours d'eau si nécessaire, relier la Montréal fortifiée à ses faubourgs (projet mis en œuvre par la démolition des fortifications). Là encore, Montréal ne faisait pas exception, car les grandes villes américaines et européennes investissaient (ou étaient en voie de le faire) dans un nouvel aménagement de leur territoire respectif ${ }^{38}$.

Les premiers projets de canalisations souterraines:

une question de salubrité urbaine?

Si les cours d'eau étaient des obstacles physiques à la libre circulation des gens et des biens, ils nuisaient aussi à la salubrité urbaine. Dès le tout début du $\mathrm{XIX}^{\mathrm{e}}$ siècle, ils étaient manifestement déjà des égouts à ciel ouvert. Les procès-verbaux des réunions tenues par les juges de paix sont clairs: les rivières de Montréal renfermaient des eaux insalubres.

Pourtant, la réglementation municipale interdisait de rejeter des eaux usées et des déchets et matières polluantes dans les cours d'eau. En 1810, on décidait que "personne ne jettera de fumier, décombre, ou ordure quelconque dans la petite rivière ou sur ses bords ${ }^{39}$ ». De même, "aucune bête morte quelconque telle que chiens, chats et autres, ne sera laissée à terre dans aucune partie de la ville ou des fauxbourgs, ni jettée dans la grande ou la petite rivière ${ }^{40} \ldots$.. En 1817 , s'ajoutait à cette interdiction celle de décharger les eaux domestiques: "Il est défendu à toutes personnes d'égouter leurs fosses d'aisance ou privé, dans la petite rivière au NordOuest de la ville, dans celles près des Sours Grises, ou dans aucun des ruisseaux qui s'y déchargent; et toutes personnes ayant actuellement aucune fosse d'aisance ou privé, qui se décharge dans les endroits susdits, seront tenues de les boucher immédiatement ${ }^{41} \ldots »$

En 1821, la réglementation municipale proscrivait de jeter des matières polluantes dans tous les cours d'eau et spécifiait un lieu de décharge sur les rives le long du Saint-Laurent ${ }^{42}$. Pourtant, les juges de paix recevaient

38. Voir en guise de synthèses, J.-L. Pinol, Le monde des villes au XIX ${ }^{\mathrm{e}}$ siècle (Paris, Hachette, 1991); E. H. Monkkonen, American Becomes Urban. The Development of U. S. Cities and Towns 17801980 (Los Angeles, University of California Press, 1988).

39. Règles et règlements de police pour la ville et les fauxbourgs de Montréal. Approuvés et confirmés par les Juges de la Cour du Banc du Roi, dans le Terme de Février, 1810 (Montréal, Imprimé par James Brown, vis-à-vis le Séminaire, 1810), article 7.

40. Règles et règlements de police pour la ville et les fauxbourgs de Montréal [...] 1810, ibid., article 10.

41. Règles et règlements de Police, pour les Fauxbourgs et la Cité de Montréal (Montréal, James Lane, 1817), article 33.

42. Règles et règlements de Police, pour la Cité et les Faubourgs de Montréal. Publiées par Autorité (Montréal, L’imprimerie de James Brown, 1821), article 21. 
encore des demandes de riverains qui désiraient tirer une conduite souterraine, de leur "privé» aux différents cours d'eau ${ }^{43}$.

Plusieurs cas recensés nous permettent d'affirmer que les eaux des rivières et des ruisseaux situés à l'intérieur de la zone urbanisée faisaient craindre pour la santé publique. En 1818, la municipalité engageait des journaliers pour nettoyer la Petite Rivière ${ }^{44}$. En 1823, les juges de paix se réunirent afin «de prévenir les effets dangereux que peuvent causer les eaux stagnantes de la rivière rue $\mathrm{Craig}^{45}$ ». L'année suivante, ils tentèrent de retracer les individus qui avaient jeté des poissons morts et autres matières jugées polluantes dans la Petite Rivière ${ }^{46}$. Les procès-verbaux des juges de paix sont remplis d'exemples semblables. En 1833, juste après l'épidémie de choléra de 1832, des résidants de la rue Wolfe (dans le faubourg Québec) craignaient pour leur santé :

Que depuis bien des années et notamment depuis quelque tems vos humbles supplians soussignés seraient extrêmement incommodés par la puanteur des eaux de la petite rivière [i.e. le ruisseau Saint-Martin] particulièrement dans l'endroit de cette rue, et qui est occasionnée par une quantité d'immondices, saloperies, paille, etc. qui sont jetées dedans et en autre des égouts de plusieurs latrines. Que les eaux de cette partie de la dite petite rivière ne s'égoutent jamais et reste a croupir ce qui cause en tout tems, ainsi que les égouts, saloperies et eaux des Tanneries de Mr Galt une puenteur insupportable surtout dans les grandes chaleurs ${ }^{47} \ldots$

43. VM-GDA, VM-35, FJPM, Procès-verbaux des Sessions spéciales de la paix, 7 avril 1821, 325. À titre d'exemple, un riverain demandait la permission d'ouvrir la rue Craig «pour y pratiquer un canal à l'effet de décharger les eaux de ses maison et emplacement dans la petite rivière ».

44. VM-GDA, VM-35, FJPM, Procès-verbaux des Sessions spéciales de la paix, 31 octobre 1818, 98-99.

45. VM-GDA, VM-35, FJPM, Procès-verbaux des Sessions spéciales de la paix, 10 juillet 1823, 285286. Les juges de paix avaient décidé par la suite de nettoyer le ruisseau, en partant de la rue Saint-Gabriel pour se rendre jusqu'au pont Prud'homme (au croisement des ruisseaux SaintMartin et Prud'homme).

46. VM-GDA, VM-35, FJPM, Procès-verbaux des Sessions spéciales de la paix, 12 juin 1824, 411. À la réunion suivante [le 26 juin 1824], on mentionnait que les diverses bêtes mortes et autres saletés qui encombraient les voies publiques avaient aussi été enlevées.

47. VM-GDA, VM-35, FJPM, Rapports et dossiers, $\mathrm{n}^{\circ}$ 3-30-20, 60 dossier-1833-9-2, "L'humble requête des propriétaires et locataires de la rue Wolfe dans le fauxbourg de Québec». Voir également pour les délibérations des juges de paix, VM-GDA, VM-35, FJPM, Procès-verbaux des Sessions spéciales de la paix, le 2 août 1833, 75-77. En 1817, les lieux de travail des activités de chapellerie et tannerie étaient pourtant bien délimités. Règles et règlements de Police, pour les Fauxbourgs et la Cité de Montréal..., op. cit., 1817, article 34: «Les chapelliers, tanneurs, ni aucune autre personne quelconque, ne pourront laver ou nettoyer en aucune saison, des peaux, chapeaux, ou autres objets relatifs à leurs métiers, dans aucune [sic] partie du canal, ou de la Grande ou Petite Rivière, excepté à l'Islette devant le port... ». 
La topographie montréalaise favorisa sans doute la transformation des rivières en égouts à ciel ouvert puisque les zones construites étaient surélevées comparativement à ces cours d'eau, jusqu'à 25 mètres dans la ville fortifiée, et de 15 à 20 mètres au bas des faubourgs de la ceinture nord. L'évacuation des eaux usées était ainsi facilitée puisque l'écoulement se faisait par gravité et ne nécessitait aucune technologie particulière.

En raison de leur dimension, la canalisation des rivières Saint-Martin, Prud'Homme et Petite Rivière en 1800 a dû nécessiter des efforts techniques et financiers considérables. Il fallut attendre la fin des années 1830 pour que les projets de canalisation des cours d'eau recueillent l'adhésion d'un plus grand nombre de partisans et qu'ils soient dorénavant considérés comme souhaitables et même réalisables. Il faut dire qu'en raison de la croissance de la population et des activités économiques, les revenus municipaux avaient augmenté depuis l'acte de 1796, ce qui levait en partie l'obstacle financier à de tels projets. La mise en œuvre de tous les projets de canalisation demanda encore des années avant d'être complétée mais ils firent à tout le moins dorénavant partie des solutions possibles pour contourner les problèmes liés à la présence de cours d'eau ${ }^{48}$.

Pour parvenir à cela s'étaient jointes aux préoccupations en matière de circulation, les considérations relatives à la salubrité urbaine. Même si celles-ci n'étaient pas nouvelles, elles prirent une importance accrue à la suite des épidémies de choléra de 1832 et de 1834. En effet, on identifia alors ces calamités à un phénomène de contagion (de là, la mise en quarantaine des populations d'immigrants). Dans le contexte de l'époque où la thèse miasmatique dominait la pensée médicale, on les associa également à un phénomène d'infection en incriminant l'environnement urbain ${ }^{49}$.

Avant les années 1830, un projet ambitieux de détournement et de canalisation de la Petite Rivière fut présenté par l'ingénieur Thomas Price $^{50}$. Ce projet datant de 1818 s'inscrivait dans le prolongement des

48. Sur la mise en place du réseau d'égouts montréalais, voir Robert Gagnon, Questions d'égouts, santé publique, infrastructures et urbanisation à Montréal au XIx $\mathrm{x}^{\mathrm{e}}$ siècle (Montréal, Boréal, 2006).

49. Pendant tout le $\mathrm{xIx}^{\mathrm{e}}$ siècle (jusqu'aux découvertes de Pasteur en fait), la théorie miasmatique selon laquelle la maladie émane des odeurs est au centre des préoccupations en matière de salubrité urbaine. C'est à Londres, avec Edwin Chadwick, que le lien entre théorie médicale de l'époque et mise en place d'infrastructures sanitaires fut illustré concrètement pour la première fois. Voir Christopher Hamlin, Public Health and Social Justice in the Age of Chadwick. Britain, 18001854 (Boston, Cambridge University Press, 1998).

50. VM-GDA, VM-35, FJPM, Rapports et dossiers, $\mathrm{n}^{\circ} 22^{\mathrm{e}}$ dossier, 1818-3, Rapport de Thomas Price, ing. civil, sur la viabilité et l'utilité de rétourner la Petite-Rivière. Il propose de recouvrir d'une rue ladite rivière, le 19 septembre 1818 . 
travaux de démolition des fortifications et avait pour principal intérêt « to prevent inundations and other nuisances to which the suburbs...». Price affirmait que son projet permettrait d'accroître la valeur des terrains situés à proximité du cours d'eau: "grater consequence the value and importance of the lands as building lots». L'ingénieur estimait ses coûts de réalisation à $12500 £$, une somme prohibitive si l'on considère que les dépenses annuelles de la municipalité à cette époque s'élevaient à un peu plus de 3000£.

Le projet ne fut pas retenu. Il confirmait néanmoins qu’il était déjà pensable pour certains - peut-être des utopistes pour leurs contemporains - d'entreprendre des travaux de canalisation des rivières dès la fin des années 1810. Jacques Viger nous apprend dans son rapport de 1825 que des riverains de la rue Craig avaient envisagé eux-mêmes, à peu près au même moment, de canaliser la rivière qui séparait cette dernière en son centre: «L'encaissement des petites rivières serait déjà fait en grande partie, surtout de celle rue Craig, par les propriétaires d'emplacemens sur cette rue, si les magistrats avaient pu accéder à leurs propositions. Ils offraient de faire toutes les avances d'argent, moyennant qu'on leur fit remise tous les ans, jusqu'à parfait paiement de leurs déboursés, du montant de leurs cotisations ${ }^{51}$. " Une fois au moins, en 1822, la proposition de détourner un cours d'eau fut mise de l'avant par les administrateurs locaux: «Résolu que l’inspecteur des chemins soit autorisé à proposer aux Ms. du Séminaire de Montréal de redresser aux frais de la ville la petite rivière passant sur leurs prairies et la rue William, aux faubourgs SaintJoseph et Sainte-Anne de manière à détourner entièrement les eaux de la dite rue William, et bâtir un pont sur la susdite petite rivière où elle traverse la dite rue de l'inspecteur - si ces messieurs veulent bien céder le terrain $^{52}$."

À la fin des années 1830, l'idée de canaliser les cours d'eau allait donc devenir à terme la solution privilégiée. En avril 1837, les juges de paix créèrent un comité pour obtenir des plans et devis pour canaliser le ruisseau Saint-Martin, situé au centre de la rue Craig ${ }^{53}$. La proposition d’alors

51. Jacques Viger, Rapports sur les chemins, rues, ruelles et ponts de la Cité et Paroisse de Montréal..., op. cit., 12-13.

52. VM-GDA, VM-35, FJPM, Procès-verbaux des Sessions spéciales de la paix, 15 juin 1822, 74-75. Le 3 août 1822, 87-90, l'Inspecteur des chemins annonçait aux juges de paix que "ces messieurs du Séminaire» donnaient leur accord au projet.

53. VM-GDA, VM-35, FJPM, Procès-verbaux des Sessions spéciales de la paix, 8 avril 1837, 79 (la question avait été abordée une première fois le 3 janvier 1837). Voir également Rapports et dossiers, $\mathrm{n}^{\mathrm{o}} 1-1837$. 
fut de construire un tunnel en brique de fort gabarit de 8 pieds sur 6 pieds (3,15 mètres sur 2,36 mètres) de la rue Saint-François-Xavier à la rue SaintLaurent, soit sur une distance d'un peu plus de 250 mètres. Le coût du projet devait être payé aux trois quarts par la ville et au dernier quart par les résidants. Aux réunions suivantes, des oppositions se dessinèrent à la table des juges de paix ${ }^{54}$, mais l'estimation des coûts du projet fut finalement déposée au mois de mai ${ }^{55}$. De toute évidence, les travaux furent réalisés rapidement car en 1840, Jacques Viger suggère de poursuivre la canalisation de la rivière, cette fois sur une distance de 380 mètres, de la rue Saint-Laurent à la rue Sanguinet.

Le premier [travail] serait à construire dans la rue Craig, et consisterait dans le prolongement (mais de moindre diamètre) du canal en brique terminant (au nord-est) à la rue St. Laurent, et qui devrait être poussé jusqu'à la rue Sanguinet. En encaissant ainsi une autre portion de la petite rivière et faisant nécessairement le remblai de son lit actuel au centre de la rue Craig, ce serait puissamment travailler à l'assainissement d'une portion considérable et populeuse de la cité, comme à son embellissement et à la jouissance de ses habitans en général, qui alors auraient une promenade de plus d'un quart de lieue de longueur sur 80 pieds de large, et qu'on pourrait complanter d'arbres ou rendre autrement agréable ${ }^{56}$.

Dans le même rapport destiné aux nouveaux élus locaux, Viger révèle que d'autres projets avaient débuté mais qu'ils nécessitaient le concours financier du pouvoir colonial. Viger marque bien l'importance maintenant accordée à la question de santé publique dans les projets de canalisation souterraine en faisant intervenir le savoir médical.

Le second canal ou égout public demandé, est également un travail important et très-coûteux, que les Magistrats ont commencé en 1837, mais n'ont point continué: c'est le canal souterain en pierre, destiné à l'écoulement des eaux des parties basses du faubourg Québec, en deçà de la Place de la Reine (anciennement Place Papineau), et, par suite, à l'assainissement d'un grand local, percé de plusieurs rues, presqu'impraticables dans ce moment, et toujours mauvaises le restant de l'année. La ville a dépensé en 1837, une assez forte somme pour ouvrir, sous la rue Lachauchetière, le solide canal en question, qui prenant du

54. VM-GDA, VM-35, FJPM, Procès-verbaux des Sessions spéciales de la paix, 15 avril, 83 et 29 avril 1837.

55. VM-GDA, VM-35, FJPM, Procès-verbaux des Sessions spéciales de la paix, 6 mai 1837, 93. Malheureusement, nous n'avons pas pu retrouver cette estimation des coûts.

56. Jacques Viger, Rapports sur les chemins, rues, ruelles et ponts de la Cité et Paroisse de Montréal..., op. cit., 10-12. 
tunnel construit aux frais de la province, sous la Place de la Reine, se prolonge de quelques cents pieds seulement sure le sud-ouest (vers la Ville), mais non pas assez loin pour recevoir toutes les eaux qui inondent les lieux en deçà. Ce commencement de canal n'est donc encore presque d'aucune utilité; mais si les travaux en étaient repris (comme il était originairement en contemplation d'en faire une partie par chaque année), et s'ils étaient amenés à la Rue de la Visitation l'intérêt public et privé y trouveraient assurément leur compte. C'est une amélioration demandée par le public depuis longtemps, conseillée à plusieurs reprises par les médecins de la cité, et l'une des plus importantes que je connaisse sous une infinité de rapports ${ }^{57}$.

En 1843, l'intervention du médecin Pierre Beaubien, alors conseiller municipal, illustre la place que prenait déjà la dimension médicale dans le domaine des infrastructures urbaines et annonçait en quelque sorte la profession de médecin hygiéniste à Montréal, qui apparaîtra dans le dernier quart du XIx ${ }^{\mathrm{e}}$ siècle. Le 15 février, il prononce un discours sur le thème des «Améliorations de la cité » à une réunion du Conseil de ville. Il y fait notamment la promotion de la canalisation du ruisseau Saint-Martin.

Les propos de Beaubien, repris quelques jours plus tard par le journal La Minerve ${ }^{58}$, témoignent de l'apparition d'une nouvelle sensibilité depuis la fin des années 1830. À l'égard de la gestion des cours d'eau, les préoccupations en matière de salubrité urbaine accompagnaient maintenant les considérations relatives à la circulation. Il faut dire que Beaubien avait séjourné une douzaine d'années en Europe ${ }^{59}$. Les influences étrangères auxquelles il avait été soumis ont sans doute contribué à orienter les propositions de ce médecin politicien.

Dans son discours, Beaubien abordait toute une série de questions, allant de l'avenir de la ville à la vitalité de son commerce, sa croissance ainsi que la salubrité et la santé de ses habitants. Au nom de la salubrité, il demandait l'ouverture de nouvelles rues plus larges, la prolongation et l'élargissement de celles existantes, la construction de places publiques, la plantation d'arbres, l'assèchement des terres basses, la construction d'égouts. À propos de la rue Craig (l'actuelle rue Saint-Antoine, alors séparée en son centre par le ruisseau Saint-Martin), Beaubien affirmait:

57. Jacques Viger, Rapports sur les chemins, rues, ruelles et ponts de la Cité et Paroisse de Montréal..., idem.

58. La Minerve, le 23 février 1843.

59. Jacques Bernier, «Pierre Beaubien», Dictionnaire biographique du Canada (Sainte-Foy, Les Presses de l’Université Laval, 1982), 11 (1881-1890): 63. Beaubien a étudié et travaillé à Paris et aurait voyagé en Allemagne, en Suisse et en Italie. 
[...] la rue Craig, [qui] devrait être ouverte jusqu'aux limites du côté Est de la ville, si en directe l'admet. Son égout couvert, ou converti en tonnelle, ses côtés bordés de nos beaux arbres forestiers, d'érables surtout; alors d'une place infecte, dégouttante, d'où s'élèvent des miasmes méphitiques, pendant vos étés chauds, vous en faites un superbe boulevard intérieur, un embellissement de votre ville, qui répandra la santé dans ses environs, et qui vous tiendra de lieu de places publiques, ou squares, que déjà vous ne pouvez plus vous procurer dans l'intérieur de la ville.

Dans le même ordre d'idée, il mentionnait également qu'il fallait porter une attention particulière aux endroits bas, marécageux, situés près du Champs de Mars, à la limite des anciens faubourgs Québec et SaintLaurent et entre les anciens faubourgs Saint-Antoine et Saint-Joseph:

Nous ne devons pas oublier que si, pendant les chaleurs de nos été, notre jeune population, notre population enfantine est décimée d'une manière si effrayante, c'est que les vents Nord-Ouest, Nord, et Nord-Est, au lieu de nous apporter un air vivifiant, souvent nous apportent, en passant sur les marécages qui viennent d'être indiqués des miasmes qui tendent beaucoup à favoriser sa destruction.

Beaubien soutenait également la réalisation de parcs et places publiques et l'élargissement des rues, d'abord afin d'aider à lutter contre les incendies, ensuite parce qu'en hiver l'accumulation de la neige y causait d'importants problèmes et enfin parce qu'en été, une chaleur excessive s'y développait $^{60}$. En fait, plus qu'un programme de salubrité urbaine, c'était l'esquisse d'une pensée urbaine, où Montréal était appelée à occuper la place centrale, qu'il présentait.

Qui peut dire ce que sera notre ville, ou le rôle qu'elle est appelée à jouer dans l'avenir. Qui peut dire ce que sera notre ville dans cinquante, dans cent ans d'ici, au centre d'un cercle de population dont tous les rayons la conduisent chez elle?

Dans les mois qui suivirent l'intervention de Beaubien, les travaux de canalisation du ruisseau Saint-Martin se poursuivirent. En 1845, la rue était élargie à 80 pieds, soit un peu plus de 30 mètres (au lieu des 30 pieds prescrits par la loi de 1796 - environ 12 mètres) ${ }^{61}$.

60. Rappelant le taux élevé de mortalité infantile, Beaubien mentionnait que selon certaines personnes, l'augmentation de la population était expliquée «non pas par la naissance des enfants de ses habitants, mais par l'établissement des habitants du dehors, qui viennent s'y fixer à un certain âge".

61. VM-GDA, VM-1, FCVM, Procès-verbaux du Conseil municipal, R.3082, bobine $25, \mathrm{n}^{\text {os }} 14,15$, 16. 


\section{LES EAUX DE PLUIE ET DE FONTE DES NEIGES}

Avec l'accroissement de la présence humaine, les eaux de pluie et de fonte des neiges commencèrent à être considérées dangereuses pour la santé humaine. Ruisselantes, elles se répandaient partout sans contrôle, elles recueillaient, charriaient et retenaient sur le territoire les déchets produits par l'activité humaine. Mais au premier chef, ces eaux pouvaient nuire à la libre circulation des gens et des biens, voire l'entraver. À Montréal, comme ailleurs dans les grandes villes occidentales ${ }^{62}$, le désir de contrôler ces eaux répondait d'abord à des impératifs de circulation auxquelles se sont additionnées des préoccupations en matière de salubrité urbaine.

Des obstacles à franchir ou comment maîtriser

l'écoulement de ces eaux qui tombent du ciel

Quand le sol était saturé ou quand, en raison de sa composition, il était imperméable, des quantités importantes d'eau pouvaient être retenues à la surface pendant des jours, voire des semaines. Elles devenaient en certains cas des obstacles quasi infranchissables ou affectaient la circulation en raison des ruptures, crevasses, glissements et affaissements qu'elles produisaient sur les rues. Nombreuses à l'époque, les rues non pavées étaient encore plus vulnérables que les rues pavées (en pierre) qui pouvaient accroître les problèmes de ruissellement de l'eau et d'inondations en raison de leur imperméabilité. Les rues non pavées (en terre ou au macadam), aussi mal conçues que les rues en pierre, pouvaient retenir l'eau bien qu'elles aient été en principe perméables. Cela occasionnait alors la formation de trous, l'accumulation de boue et l'enlisement des charrettes et chariots.

L'écoulement et, à terme, l'évacuation des eaux de pluie et de la fonte des neiges impliquaient toute une série d'activités complémentaires de terrassement pour remodeler le territoire: creusage de caniveaux ou fossés (rigoles) en bordure des rues, nivellement et aplanissement des buttes, monticules et promontoires, rehaussement ou abaissement des niveaux du sol. En fait, maîtriser ces eaux de surface qui tombent du ciel nécessitait un jeu d'équilibre complexe qui consistait à mettre à niveau les rues de la ville, afin de compenser ici pour une dépression, de contrebalancer là pour rediriger l'eau, d'accentuer ailleurs une pente pour accroître l'apport d'eau, etc. Le terrassement à entreprendre nécessitait deux étapes à la fois distinctes et complémentaires. D’abord, il s'agissait d'aplanir la rue qui avait été construite sans «correctif » de la surface du sol, en épousant

62. Voir notamment Sabine Barles, La ville délétère..., op. cit. 
ses multiples vallons (plus ou moins hauts et plus ou moins étendus). Ensuite, il était nécessaire de mesurer les hauteurs des rues à l'échelle du territoire urbanisé en entier afin de trouver un équilibre, en quelque sorte, entre les unes et les autres, d'une part, et entre ces rues et le cadre bâti, d'autre part. C'était donc un travail rompu à la "micro-topographie», d'une part, et à la «macro-topographie», d'autre part ${ }^{63}$.

La loi de 1796 concédait aux juges de paix des pouvoirs en matière d'eau, nous l'avons déjà mentionné. À ceux-ci s'ajoutent en 1799 des pouvoirs en matière de terrassement afin de pouvoir corriger, au besoin, l'environnement urbain:

Et il sera du devoir de tel Inspecteur [des chemins], avant de procéder à l'aplanissement, élévation ou pavé d'aucune Rue, Ruelle ou Place publique, ou à l'ouverture d'aucun Canal, Cours d'Eau ou Aqueduc, ou à l'érection d'aucun Pont ou Chaussée dans les dites cités et paroisses de Québec et Montréal, de dresser un Plan d'icelle Rue, Ruelle ou Place publique, Canal, Cours d'Eau ou Aqueduc, Pont ou Chaussée, représentant leur niveau et déclivité, accompagné d'un Procès Verbal au dit plan, sur le mode le plus convenable et expédient pour l'exécution des ouvrages y proposés, lequel plan et Procès verbal seront déposés en l'Office du Greffier de la Paix de chacun des Districts de Québec et de Montréal respectivement, et notice sera donné en la manière que les Juges à Paix trouveront convenable, aux propriétaires de terreins ou maisons, ou autres bâtiments joignant telle Rue, Ruelle, Place publique, Canal, Cours d'eau, Aqueduc, Pont ou Chaussée, et autres intéressés, que tel plan et Procès verbal sont ainsi déposés pour leur inspection gratuite, à ce qu'ils ayent à faire, tous un délai qui n'excédera pas un mois, leurs observations et oppositions si aucunes ils ont au contraire, pour y être fait droit, sinon, les dits plans et Procès verbaux être [seront] homologués, et ensuite exécutés selon leur forme et teneur ${ }^{64}$.

L'objectif de l'Inspecteur des chemins, bien que complexe à réaliser, consistait, d'une part, à mettre en place un circuit ou un tracé d'écoulement hiérarchisé (jusqu'aux déversements dans les cours d'eau réceptacles - le fleuve Saint-Laurent et les ruisseaux) et, d'autre part, à ne pas favoriser par son propre travail la formation de rues ou de zones inondables. Il devait ainsi se prêter à un véritable jeu d'équilibriste.

63. William Cronon, Nature's Metropolis. Chicago and the Great West (W. W., New York, Norton and Company, 1991), 58. W. Cronon a déjà montré pour le cas de Chicago comment on en était venu là-bas à décider d'élever la ville afin de remédier aux problèmes d'écoulement de l'eau qui affectaient la circulation et à terme le commerce.

64. Statuts du Bas-Canada, 39 Geo III (1799) chapitre 5, article 26. 
Concrètement, voici comment se présentait le travail à entreprendre à l'échelle d'un quadrilatère de rues: «Résolu [...] d'élever cette partie de la rue du Collège depuis la rue Ste. $\mathrm{H}[s i c]$ jusqu'à la rue McGill et ladite rue McGill depuis la pont Charland jusqu'à la rue St. Paul de façon a égoutter les eaux dans des grilles à placer à l'encoignure de la dite rue St. Paul avec celles McGill et du Collège ${ }^{65}$. " La figure suivante (figure 3) offre une vision schématique de l'exercice qu'il lui fallait effectuer. Les flèches indiquent la direction que devait emprunter l'eau pour se rendre dans une grille.

Ces grilles (des bouches d'égouts en fait) donnaient sur une ouverture pratiquée dans les conduites d'égout dont quelques rues étaient munies à l'époque: "Résolu que partout où il sera fait des canaux ou égouts publics sous aucune des rues de la cité, l'année prochaine, il soit aussi fait des canaux perpendiculaires - grilles en fer pour la décharge de l'eau des rues transversales ${ }^{66} . »$

À l'échelle encore plus réduite de la rue en particulier, le travail de terrassement pour maitriser les eaux de pluie et de fonte des neiges était tout aussi complexe et nécessitait du savoir-faire. Il s'agissait de substituer à un environnement à la surface inégale, accidenté, par trop naturel dans ses caractéristiques topographiques, un environnement naturalisé, reconfiguré, retravaillé, uniformisé dans ses parties. Ce premier travail consistait à niveler le sol et le mettre à niveau, ce qui n'était pas sans causer bien des oppositions de la part des propriétaires de bâtiments. En effet, ces travaux étaient sources de litiges ${ }^{67}$ car le cadre bâti avait été construit en épousant la topographie naturelle (avec ses buttes, ses vallons et ses dépressions), laquelle, une fois les travaux de nivellement et de mise à niveau complétés, présentait un tout nouveau portrait: un portrait qui refusait toute partie accidentée et de trop fortes dépressions. Certains propriétaires fonciers voyaient ainsi les fondations de leurs bâtiments se retrouver plusieurs centimètres (peut-être plus d'un mètre, même) sous le niveau de la rue. Par exemple, on annonce en 1834 que le niveau de la rue Sainte-Élisabeth

65. VM-GDA, VM-35, FJPM, Procès-verbaux des Sessions spéciales de la paix, 1 mai 1835, 45.

66. VM-GDA, VM-35, FJPM, Procès-verbaux des Sessions spéciales de la paix, 23 décembre 1822, 120.

67. Statuts du Bas-Canada, 36 Geo III (1796) chapitre 9, articles 45 et 46. Dès 1796, des procédures en cas de litiges entre des propriétaires et l'administration locale avaient été édictées par le législateur. La cause était portée devant un corps de jurés - composé de résidants du quartier - qui avaient à décider s'il y avait des modifications à adopter au projet ou des compensations financières à verser. Par ailleurs, il semble bien que ce corps de jurés n'avait pas la capacité de remettre en cause la réalisation des projets. 
Figure 3

L'écoulement de l'eau en direction d'égouts souterrains

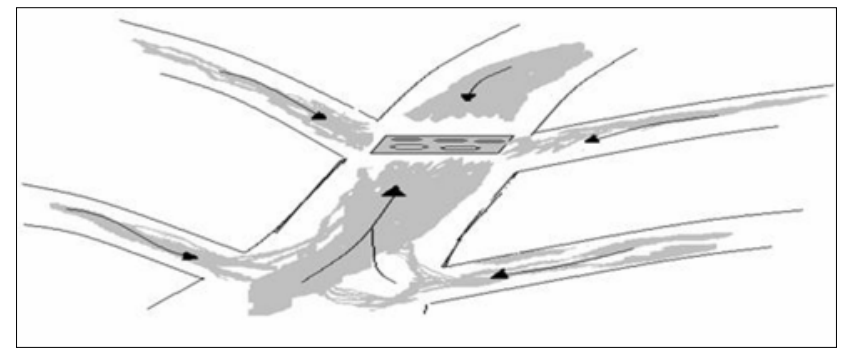

Réalisation: Dany Fougères, 2006.

sera haussé d'au moins 18 pouces (46 centimètres). Ailleurs, des propriétaires, qui comptaient éventuellement subdiviser et développer leurs terrains encore en friche, constataient que le haussement du niveau des rues imposerait des travaux de remblaiement avant les mises en chantier.

Les changements de niveaux étaient assez importants en plusieurs endroits pour que Jacques Viger demande, dans son rapport de 1825 , qu'on puisse obliger les propriétaires de terrains à clôturer ces derniers (en fait pratiquer des murs de soutènement) afin de prévenir les éboulements: «Cependant, faute de pouvoir exiger de pareilles clôtures sur les rues que la ville fait niveler à Montréal, où le sol est si différent de celui de Québec, on conçoit combien ces sortes de travaux se peuvent aisément et vite détériorer, soit par les éboulements sur la voie publique des terrains plus élevés qu'elle, soit par ceux de cette même voie sur les terrains plus bas qu'elle ${ }^{68}$. Pourtant, les règlements municipaux de 1817 étaient clairs à cet égard, les propriétaires devaient clôturer leur propriété : «Tous propriétaires ou occupants de terreins ou emplacemens dans la Ville ou les Fauxbourg, seront tenus de les clorre, ou faire clorre de tout les côtés, où tels terreins ou emplacemens joindront une place publique, rue, ruelle ou chemin, avec un mur de pierre ou de briques, ou une palissade assise sur une fondation des mêmes matériaux ${ }^{69} \ldots$ ”

68. Jacques Viger, Observations et amélioration des Lois des Chemins..., op. cit., 12. Retrouver sa propriété sous le niveau de la rue pouvait occasionner des problèmes majeurs, dont celui d'être continuellement inondé par l'eau provenant de la rue. Inversement, le fait d'avoir les fondations de sa propriété au-dessus du niveau de la rue ne causait pas de problème majeur, sinon celui de devoir s'assurer qu'il n'y ait pas de glissement de terrain. Comme on peut le comprendre à la lecture de Jacques Viger, le propriétaire évitera cela en construisant un muret ou une clôture. Manifestement, les propriétaires dont le terrain était surélevé à la suite des travaux de mise à niveau étaient peu enclins à payer ces coûts.

69. Règles et règlements de Police, pour les Fauxbourgs et la Cité de Montréal..., op. cit., 1817, article 3. 
Manifestement, ce règlement était peu ou pas suivi encore en 1825. La figure 4 présente un schéma qui permet de se représenter de manière simplifiée l'ampleur de la tâche à laquelle devait faire face l'inspecteur des chemins pour niveler et mettre à niveau les rues. Elle montre aussi les problèmes que pouvaient soulever ces mêmes travaux pour les propriétaires riverains. Le trait en gras illustre la rue une fois les travaux de nivellement et de mise à niveau complétés; le trait fin illustre la topographie naturelle avant les travaux.

Ce travail, colossal à l'époque si l'on tient compte des moyens techniques mis à la disposition des maîtres d'œuvre, avait le double avantage d'améliorer les conditions de déplacement et le contrôle des eaux urbaines. Mais attention, on ne pouvait se permettre de faire de la rue une immense cuvette pour répondre aux objections des riverains dont les propriétés étaient les plus basses et on ne pouvait pas se permettre non plus de hausser le niveau de la rue au point où ces mêmes riverains auraient vu disparaittre à l'horizon la moitié verticale de leur propriété.

À partir du milieu des années 1820, un autre procédé, complémentaire à la mise à niveau des rues fut aussi employé afin de préserver l'intégrité physique des rues et de prévenir l'accumulation d'eau (figure 5). La rue bombée, qui assure l'écoulement des eaux de part et d'autre des deux côtés des rues, remplaça graduellement la rue plate ${ }^{70}$. Les travaux visaient à soulever le centre de la rue. L'eau s'écoulait dans les rigoles, caniveaux ou fossés creusés à cet effet au lieu d'y demeurer plusieurs jours.

En 1840, Jacques Viger mentionnait dans son rapport sur les rues de Montréal que ce travail de reconfiguration n'était pas encore complété pour l'ensemble des rues existantes. À titre d'exemple, il précisait, au sujet de la rue Saint-Denis, qu'il fallait «former le centre en l'arrondissant au milieu par fossés de chaque côté ${ }^{71} »$.

À ces travaux de terrassement visant à contrôler les eaux de surface s'ajoutait aussi l'activité réglementaire des autorités locales ${ }^{72}$. En effet, les Montréalais étaient tenus de contrôler les eaux de pluie et de fonte des neiges qui s'accumulaient sur les toits de leurs résidences pour ensuite s'égoutter le long des façades jusqu'à la rue:

70. Voir Dany Fougères, «Des rues et des hommes...», op. cit.

71. Jacques Viger, Rapports sur les chemins, rues, ruelles et ponts [...] 1840, op. cit., 6-7.

72. Sur le plan de l'action réglementaire, Montréal et la ville de Québec présentaient plusieurs similitudes. Sur Québec, voir J. Hare, M. Lafrance et D.-T. Ruddel, Histoire de la ville de Québec, 1608-1871 (Montréal/Ottawa, Boréal/Musée canadien des civilisations, 1987). 
Figure 4

Le nivellement et la mise à niveau des rues

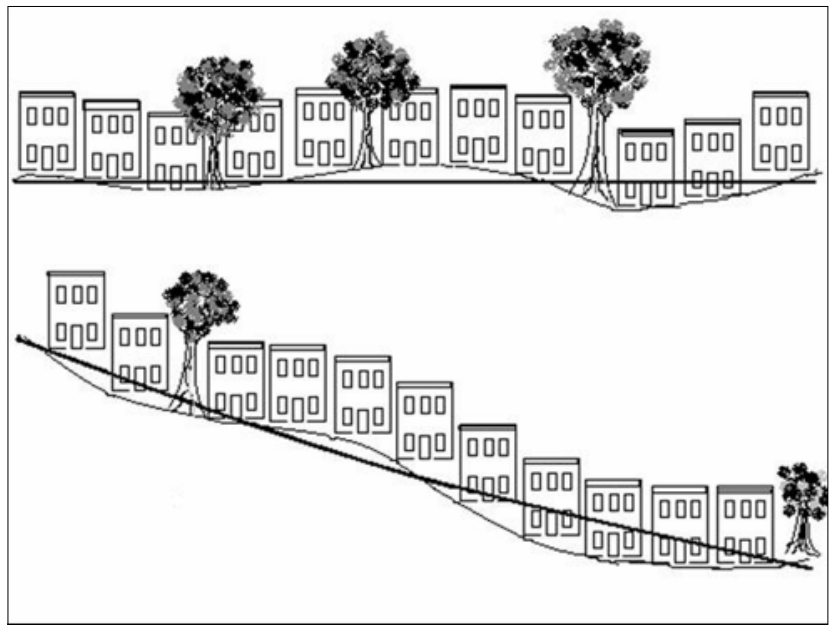

Réalisation: Dany Fougères, 2006.

L'expérience ayant prouvé que l'eau qui tombe des gouttières qui s'avancent dans les rues est fort préjudiciable au pavé des dites rues et incommode beaucoup les passants, il est ordonné que l'eau tombant de la couverture de touts bâtiments ou maisons joignant dans la ville a une rue, ruelle ou place publique et dans les fauxbourgs à une des rues qui ont été nivelées, soit conduite de la couverture dans des gouttières couvertes qui descendront le long des dits bâtiments ou maisons jusqu'à un pied de terre ${ }^{73} \ldots$

Autre exemple de l'action normative locale, les Montréalais furent tenus de participer activement à la lutte pour le contrôle des eaux sur l'espace public à partir de 1821: "Toutes les fois qu'il se formera des mares dans aucunes des rues, ruelles, ou places publiques, de la ville ou des faubourgs, pendant l'hiver ou le printems, tous propriétaires ou occupants de maisons ou terrains, devant lesquels se trouveront telles mares, seront tenus de les égouter par des rigolles suffisantes ${ }^{74}$.» Ici, il est possible d'imaginer les difficultés rencontrées par les dirigeants locaux pour s'assurer du respect d'une telle directive. Il était certainement plus facile de prendre en défaut

73. Règles et règlements de police pour la ville et les fauxbourgs de Montréal [...] 1810, ibid., article 21; Règles et règlements de Police..., 1817, op. cit., article 16; Règles et règlements de Police..., op. cit., 1821, article 32 .

74. Règles et règlements de Police..., op. cit., 1821, article 20. 
Figure 5

La reconfiguration géométrique des rues



Réalisation: Dany Fougères, 2006.

quelqu'un qui n'avait pas installé des gouttières; dans ce cas-là, il était difficile d'argumenter devant le constat d'infraction.

Si la réglementation ainsi que tous ces travaux de nivellement, de mise à niveau et de reconfiguration des rues permettaient d'accroître la maîtrise des eaux urbaines, encore fallait-il que ces dernières puissent être recueillies puis évacuées. Comme la figure précédente (figure 5) le montre, ces eaux s'écoulaient dans des caniveaux, des fossés, des rigoles (à ciel ouvert) ou à l'intérieur d'égouts pluviaux (souterrains). Ces ouvrages recueillaient les eaux qui, pour une partie, pouvaient pénétrer dans le sol (les caniveaux, fossés et rigoles n'étaient pas des ouvrages imperméables) et qui, pour une autre partie, s'écoulaient jusqu'au fleuve notamment par une succession de trajets (à ciel ouvert et souterrains) en dépression les uns à la suite des autres. Montréal profitait ici de sa proximité au fleuve, avantage qui n'était pas donné à toutes les villes canadiennes ${ }^{75}$.

\section{Ces eaux qui «charrient» la maladie}

Le terrassement des rues, par son objectif d'assurer l'écoulement de l'eau à des fins de libre circulation, permettait également d'éviter la formation

75. Certaines villes durent composer avec un territoire plat, où l'évacuation des eaux constitua un défi technique important. Voir notamment N. R. Ball, dir, Bâtir un pays..., op. cit.; pour sa part, Toronto bénéficiait de sa proximité à une grande étendue d'eau. Voir Catherine Brace, «Public Works in the Canadian City; the Provision of Sewers in Toronto 1870-1913", Urban History Review/Revue d'histoire urbaine, 23,2 (mars, 1995): 34-43. 
de cloaques, chargées de déchets et de matières fécales, se retrouvant un peu partout sur le territoire urbanisé. Les préoccupations en matière de salubrité urbaine ou, autrement dit, en matière de propreté de la ville, allaient donc s'ajouter à l'argumentaire en faveur de la configuration des rues.

Ces préoccupations étaient évidemment antérieures aux travaux de terrassement des rues qui eux s'accélèrent à partir des années 1820. En effet, la loi de 1796 prévoyait déjà que les propriétaires des fossés et caniveaux donnant sur des propriétés privées devaient les nettoyer ${ }^{76}$. Dès 1810 , les règlements municipaux prescrivaient le nettoyage des rues et le ramassage des ordures aux frais du public:

Tous ceux qui occupent des maisons ou emplacements sur les places, rues ou passages de la ville ou des fauxbourgs, où il y a des pavés ou des gouttières [fossés, caniveaux], mettront l'ordure en tas devant leurs maisons ou emplacements, depuis le premier de Mai jusqu'au quinze de Novembre de chaque année le Samedi de chaque semaine [...]. Dans les rues ou l'on a fait des gouttières, les dites gouttières seront nettoyées par les propriétaires ou occupants des maisons ou emplacements, et les ordures ou décombres seront mis en tas sur les côtés des rues près des gouttières, et ne seront étendus sur aucune partie des dites rues pour aucune raison quelconque [...] et les dits décombres et ordures seront emportés le même jour, aux frais publics, par des charetiers [...]; et les occupants de maisons et emplacements sur les rues St. Charles et la Fabrique et sur la place de l'ancien marché seront tenus de ballayer et mettre en tas les ordures comme susdit, seulement jusqu'au milieu des dites rues et au milieu de l'espace compris entre les maisons et les étaux sur chacune des dites places; et tous charetiers qui seront ainsi employés, pourront se plaindre de toute personne qui manquera de se conformer au présent règlement ${ }^{77} \ldots$

En 1817, les règlements étaient encore plus explicites: les personnes dont la résidence donnait sur une rue pavée devraient balayer celle-ci; dans les rues non pavées, les riverains devraient nettoyer les fossés ${ }^{78}$.

Toutefois, les règlements municipaux n'étaient pas toujours suivis à la lettre. En voici deux exemples, le premier datant de 1823 et le second de 1834: «Résolu que l'Inspecteur soit autorisé à employer huit voitures et dix huit journaliers, si nécessaire, pour nétoyer les égouts de diverses rues

76. Statuts du Bas-Canada, 36 Geo III (1796) chapitre 9, article 43.

77. Règles et règlements de police pour la ville et les fauxbourgs de Montréal [...] 1810, op. cit.., article 23 .

78. Règles et règlements de Police..., 1817, op. cit., article 18. 
de la Cité, et consolider où besoin, et ce jusqu'à nouvel ordre ${ }^{79}$ »; «[Résolu de] fossoyer, arrondir et remplir les ornières des rues non pavées, partout où il [l'inspecteur des chemins] jugera nécessaire de le faire ${ }^{80}$ ».

Ces solutions techniques, la mise à niveau des rues, leur succession en dépression les unes par rapport aux autres, le creusage de fossés et de caniveaux et l'introduction de la rue bombée en son centre, rendaient possible la mise en œuvre d'une nouvelle "pièce maîtresse» en matière de salubrité urbaine : l'arrosage des rues ${ }^{81}$. En effet, elles permettaient de répandre des eaux de «lessives» sur les chaussées (pavées). Le même procédé était d'ailleurs utilisé dans d'autres grandes villes occidentales, à Paris notamment, comme le souligne $S$. Barles ${ }^{82}$. Dans les rues non pavées de Montréal (qui pour la plupart étaient construites en macadam), on se contentera de balayer et de ramasser les ordures puis de nettoyer les fossés pour les garder libres.

Ainsi au début des années 1840, les eaux qui tombaient du ciel étaient dorénavant "capturées» et orientées à l'intérieur de tout un circuit de caniveaux, fossés et rigoles, pour ensuite être évacuées et rejetées dans les rivières intérieures et, au mieux, jusqu'au fleuve Saint-Laurent. Ces eaux ne seront définitivement "cachées» de la vue de tout un chacun qu'au moment où la municipalité se lancera dans la construction d'un vaste réseau d'égouts étendu à l'échelle de la ville en entier. Avant 1840, la pose des conduites d'égouts pluviaux se fit sans plan d'ensemble ${ }^{83}$. Au gré des actions individuelles, un usage sanitaire en fut peu à peu développé. En effet, après avoir obtenu la permission des juges de paix, des propriétaires raccordaient à leurs frais leur "privé» aux égouts municipaux ${ }^{84}$. La réalisation d'un réseau d'égouts souterrains (accueillant à la fois les eaux pluviales et sanitaires) ne sera complétée à l'échelle du territoire mont-

79. VM-GDA, VM-35, FJPM, Procès-verbaux des Sessions spéciales de la paix, 31 mai 1823, 245.

80. VM-GDA, VM-35, FJPM, Procès-verbaux des Sessions spéciales de la paix (Conseil de ville), (n.d.) 1834,7 .

81. Voir Dany Fougères, L'approvisionnement en eau à Montréal..., op. cit., 200.

82. Elle écrit: «Ce système [d'arrosage des rues] ne peut fonctionner pleinement qu'avec la modification du profil des rues et le passage, dans les années 1830, des chaussées fendues aux chaussées bombées qui comprennent deux ruisseaux latéraux (par opposition à l'unique ruisseau central fendu) et dont les ingénieurs louent l'efficacité en termes d'assainissement et de circulation ». Sabine Barles, La ville délétère..., op. cit., 212.

83. Voir Dany Fougères, L'approvisionnement en eau..., op. cit., 189-192.

84. Mentionnons tout de même que cet usage était marginal, uniquement pour les propriétaires ayant chez eux des «lieux à l'anglaise», étant donné l'absence d'équipements domestiques sanitaires (raccordés à l'eau courante) dans la première moitié du XIX ${ }^{\mathrm{e}}$ siècle. 
réalais qu'à la toute fin du $\mathrm{xIX}^{\mathrm{e}}$ siècle ${ }^{85}$. Avant 1840 , il s'agissait avant tout de mettre en place des infrastructures pour recueillir les eaux de pluie et ensuite les évacuer (sans traitement bien entendu). Comme c'était le cas à l'égard des cours d'eau, les rejets des eaux domestiques étaient aussi prohibés dans les fossés et les caniveaux qui, par définition, étaient des infrastructures à ciel ouvert ${ }^{86}$.

Tout égout ou goutière se déchargeant directement d'une maison ou bâtiment dans une place, rue ou passage de la ville ou des fauxbourgs, sera bouché ou ôté sous peine de vingt chelins pour toute et chaque contravention [...]. Mais comme il n'y a point d'égouts ou de cloaques publics pour conduire l'eau sous terre, ce règlement n'est pas entendu regarder comme contravention, l'eau qui coule dans aucune rue, place ou chemin d'une cour dépendante de quelque maison ou bâtiment ${ }^{87}$.

Le creusage des caniveaux des fossés et des rigoles et leur empierrement (on posait du gravier dans les extrémités les plus profondes) ainsi que la pose de conduites d'égouts (en brique ou en pierre) ${ }^{88}$ occupaient une part importante des activités menées par l'inspecteur des chemins: en fait, les travaux réalisés sur les rues seront toujours accompagnés de travaux destinés à recueillir les eaux de pluie pour en permettre ensuite l'évacuation. D’abord mis en œuvre principalement pour des préoccupations d'aménagement et de circulation comme ce fut le cas à l'égard des cours d'eau, les travaux visant à contrôler les eaux de pluie et de fonte des neiges ont graduellement adopté un objectif clair de salubrité urbaine. S’ajoute à cette mesure, dans les années 1860 , le nettoyage des rues par des eaux de lessive, rendu possible par la reconfiguration des rues et par la mise en place d'un service d'eau potable.

\section{CONCLUSION}

En 1827, un voyageur du nom de Thomas Johnston écrivait au sujet des rues de Montréal: «The streets are open and regular, compared with those of Quebec, and during the summer, kept remarkably clean ${ }^{89}$." Cette

85. Voir Robert Gagnon, Une question d'égout..., op. cit.

86. Règles et règlements de Police..., 1817, op. cit., article 15 et Règles et règlements de Police..., op. cit., 1821, articles 14 à 19 .

87. Règles et règlements de police pour la ville et les fauxbourgs de Montréal [...] 1810, op. cit.., article 6.

88. VM-GDA, VM-35, FJPM, Procès-verbaux des Sessions spéciales de la paix, 24 décembre 1822, 120-122. Les spécifications techniques des conduites d'égout qui seront à l'avenir construites sont présentées lors de cette réunion.

89. Thomas Johnston, Travels Through Lower Canada, Interspersed With Canadian Tales And Anecdotes, And Interesting Information To Intending Emigrants (Edinburgh, J. Glass, 1827), 39. 
évaluation n'était pas partagée par plusieurs Montréalais de l'époque : les pétitions déposées à la table des juges de paix montraient des insatisfactions répétées et l'inspecteur Viger se plaignait du mauvais état des rues (voire du manque de financement pour les construire et les entretenir) dans ses rapports de 1825 et de 1840 . Une analyse comparative de l'état du parc d'infrastructures routières avec d'autres villes de taille similaire devrait éventuellement être réalisée.

Si notre voyageur était repassé quelque dix ans plus tard, nous aurait-il entretenus des moyens techniques mis de l'avant à Montréal pour résoudre les problèmes de salubrité urbaine? D’ailleurs se serait-il intéressé à cette question? Sans doute, car partout dans les grandes villes s'amorçait alors plus que jamais une phase de mise en place d'infrastructures destinées à répondre aux problèmes de salubrité urbaine. Pour une large part, cette phase faisait suite aux épidémies qu'avaient connues (à des degrés divers) les pays occidentaux.

Selon nous, l'action publique montréalaise au tout début du $\mathrm{XIX}^{\mathrm{e}}$ siècle s'inscrivait d'abord dans un objectif d'aménagement du territoire. Circulez, voilà le projet à mettre de l'avant et voilà le prisme à travers lequel la gestion de l'eau est réalisée. Lorsque la ville reçoit sa charte d'incorporation permanente en 1840, la même préoccupation d'aménagement est encore présente. Les nouveaux administrateurs locaux, élus cette fois, ont "pouvoirs et autorités [...] ayant rapport à ou concernant, la projection, la façon, érection, maintien en réparation et règlement de tous grands chemins, ponts, rues, places, ruelles, chaussées, pavés, fossés, levées, cours d'eau, égouts ${ }^{90} \ldots »$. Toutefois, la question de la salubrité urbaine se rattache définitivement à ce moment à la recherche des solutions visant à gérer les eaux urbaines. Ici, les épidémies, les croyances médicales, les capacités financières de la ville et la croissance de celle-ci, sont autant d'éléments qui s'additionnent et se conjuguent à la faveur de solutions techniques.

À partir de 1840, ces solutions techniques sont portées par une administration municipale composée d'élus qui, peu importe leur allégeance politique, cherchent tous à faire de la ville un lieu d'échange et un espace économique et à y faire croître la valeur des capitaux et des biens fonciers, y compris les leurs. Pour se réaliser, cet espace économique nécessitait la construction et la mise en place d'infrastructures urbaines. Améliorer et Montréal », chapitre 36, article 43. 
faciliter les déplacements dans la ville étaient un gage de croissance économique, tout comme l'était la mise en place d'équipements pour assurer la propreté de la ville, qui devenait un lieu favorable à l'implantation des industries et à l'accueil de travailleurs. Cela dit, on aura constaté que le discours économique n’a pas suffi, à lui seul, pour introduire une nouvelle conception de la place des eaux de surface dans la ville: les questions relatives à la salubrité urbaine ont été déterminantes. 